\title{
FoxO3 regulates hepatic triglyceride metabolism via modulation of the expression of sterol regulatory-element binding protein $1 \mathrm{C}$
}

Liu Wang ${ }^{1,2+}$, Xiaopeng Zhu ${ }^{1,2+}$, Xiaoyang Sun ${ }^{1,2}$, Xinyu Yang ${ }^{1,2}$, Xinxia Chang ${ }^{1,2}$, Mingfeng Xia ${ }^{1,2}$, Yan Lu ${ }^{1,2}$, Pu Xia ${ }^{1,2}$, Hongmei Yan $^{1,2^{*}}$, Hua Bian ${ }^{1,2^{*}}$ and Xin Gao ${ }^{1,2}$

\begin{abstract}
Background: Excessive intrahepatic lipid accumulation is the major characteristic of nonalcoholic fatty liver disease (NAFLD). We sought to identify the mechanisms involved in hepatic triglyceride (TG) homeostasis. Forkhead box class $\mathrm{O}$ (FoxO) transcription factors have been shown to play an important role in hepatic metabolism. However, little is known about the effect of $\mathrm{FoxO} 3$ on hepatic TG metabolism.

Methods: Liver biopsy samples from patients with NALFD and liver tissues from high glucose and high sucrose (HFHS) fed mice, ob/ob mice and db/db mice were collected for protein and mRNA analysis. HepG2 cells were transfected with small interfering RNA to mediate $\mathrm{FoxO} 3$ knockdown, or adenovirus and plasmid to mediate FoxO3 overexpression. FoxO3-cDNA was delivered by adenovirus to the liver of C57BL/6 J male mice on a chow diet or on a high-fat diet, followed by determination of hepatic lipid metabolism. Sterol regulatory element-binding protein 1c (SREBP1c) luciferase reporter gene plasmid was co-transfected into HepG2 cells with FoxO3 overexpression plasmid.

Results: FoxO3 expression was increased in the livers of HFHS mice, ob/ob mice, db/db mice and patients with NAFLD. Knockdown of FoxO3 reduced whereas overexpression of FoxO3 increased cellular TG concentrations in HepG2 cells. FoxO3 gain-of-function caused hepatic TG deposition in C57BL/6 J mice on a chow diet and aggravated hepatic steatosis when fed a high-fat diet. Analysis of the transcripts established the increased expression of genes related to TG synthesis, including SREBP1C, SCD1, FAS, ACC1, GPAM and DGAT2 in mouse liver. Mechanistically, overexpression of FoxO3 stimulated the expression of SREBP1C, whereas knockdown of FoxO3 inhibited the expression of SREBP1c. Luciferase reporter assays showed that SREBP1c regulated the transcriptional activity of the SREBP1C promoter.
\end{abstract}

Conclusions: FoxO3 promotes the transcriptional activity of the SREBP1c promoter, thus leading to increased TG synthesis and hepatic TG accumulation.

Keywords: Nonalcoholic fatty liver disease, NAFLD, Forkhead box class O3, FoxO3, Sterol regulatory elementbinding protein1c, SREBP1C

\footnotetext{
*Correspondence: yan.hongmei@zs-hospital.sh.cn; bianhuaer@126.com; zhongshan_bh@126.com

† Liu Wang and Xiaopeng Zhu should be considered co-first authors

'Department of Endocrinology and Metabolism, Zhongshan Hospital, Fudan

University, Shanghai 200032, China

Full list of author information is available at the end of the article
}

C The Author(s). 2019 Open Access This article is distributed under the terms of the Creative Commons Attribution 4.0 International License (http://creativecommons.org/licenses/by/4.0/), which permits unrestricted use, distribution, and reproduction in any medium, provided you give appropriate credit to the original author(s) and the source, provide a link to the Creative Commons license, and indicate if changes were made. The Creative Commons Public Domain Dedication waiver (http://creativecommons.org/publicdomain/zero/1.0/) applies to the data made available in this article, unless otherwise stated. 


\section{Introduction}

Nonalcoholic fatty liver disease (NAFLD) is the predominant cause of chronic liver disease. The incidence of NAFLD in the world is $25.24 \%$, with a range of $13.5 \%$ in Africa to $31.8 \%$ in the Middle East [1]. NAFLD is a highly prevalent metabolic disease closely linked to insulin resistance and metabolic syndrome, leading to an increased risk of liver cirrhosis and hepatocellular carcinoma, type 2 diabetes mellitus, cardiovascular diseases, and chronic kidney disease [2]. The pathogenesis of NAFLD has been extensively studied but remains poorly understood. Disturbed lipid homeostasis and an excessive accumulation of triglyceride (TG) and other lipid species is the first step in the pathophysiology of NAFLD. Insulin resistance, enhanced de novo lipogenesis (DNL), and a high-fat diet are pivotal for the development of hepatic steatosis [3, 4].

Forkhead box class $\mathrm{O}$ (FoxO) is a nuclear protein subfamily that includes four homologous proteins in mammals: FoxO1, FoxO3, FoxO4 and FoxO6. These proteins share a highly conserved Forkhead DNA binding domain [5]. FoxOs mediate the inhibitory actions of insulin or insulin-like growth factor on key genes in diverse pathways that include cell cycle regulation, energy metabolism, proteostasis, oxidative stress, apoptosis and immunity [5-9]. Current studies characterized FoxO1 as an important regulator of gluconeogenic activity and lipid metabolism [10]. FoxO3 has the highest degree of homology in amino acid sequence with that of FoxO1 [11], in accordance with mild hepatic glucose production [12]. In lipid metabolism, the homolog of FoxO3 in C. elegans, DAF-16, enhanced the expression of gene networks involved in lipid synthesis [13]. However, little is known about the role of FoxO3 in lipid metabolism in mammals. Two cell experiments showed that palmitic acid (PA) or stearate treatment upregulated nuclear FoxO3 protein $[14,15]$. Consistently, our team found that FoxO3 expression was elevated in the livers of rats fed a high-fat diet via microarray analysis and confirmed by real-time PCR (Additional file 1: Figure S1a). In human studies, there was a correlation between FoxO3 gene polymorphism and the insulin resistance index and body mass index [16]. Together, these results imply that abnormal expression of FoxO3 may be closely associated with NAFLD. Nonetheless, whether and how FoxO3 affects hepatic TG metabolism has been poorly explored.

DNL is thought to play a significant role in the pathogenesis of NAFLD, with sterol regulatory-element binding protein 1c (SREBP1c) acting as a major regulator [17]. In the context of DNL, SREBP1c is activated to promote the transcription of lipogenic genes such as fatty acid synthase (FAS), acetyl CoA carboxylase 1 (ACC1) and stearoylCoA desaturase 1 (SCD1) [18]. Here, we used two independent approaches (loss or gain-of-function) to study what and how FoxO3 exerts effects on hepatic TG metabolism in vitro and in vivo. FoxO3 knockdown suppressed whereas FoxO3 overexpression increased intracellular TG levels. A dual luciferase reporter assay showed that FoxO3 activated the SREBP1c promoter. Thus, we identified that FoxO3 prompted hepatic steatosis via transcriptionally upregulating the expression of SREBP1c.

\section{Methods}

\section{Human liver samples}

We collected eight liver biopsy samples at Zhongshan Hospital, Fudan University. There were four samples from normal subjects and four samples from patients with NAFLD diagnosed by liver biopsy. This study was in accordance with the Helsinki Declaration of 1975 and approved by the ethics committee of Zhongshan Hospital, Fudan University, and each subject provided written informed consent.

\section{Reagents and antibodies}

We obtained insulin, glucose and palmitic acid from Sigma-Aldrich. The anti-FoxO3 antibody (\#2497) was purchased from Cell Signaling Technology (Cell Signaling, USA). The anti-SREBP-1 antibody (\#8984) was purchased from Santa Cruz Biotechnology, Inc. (Santa Cruz, USA). The mouse monoclonal antibody against $\beta$-actin was purchased from Sigma (St. Louis). The goat polyclonal secondary antibodies against mouse (\#3032) or rabbit (\#3012) were purchased from Signal way Antibody (SAB, USA). The dual-luciferase reporter assay kit was purchased from Promega (Madison, USA).

\section{Cell culture}

Human HepG2 and $293 \mathrm{~T}$ cells were maintained in DMEM (Gibco, USA) containing 1\% penicillin/streptomycin (Invitrogen, USA), $1 \mathrm{~g} /$ liter glucose, and 10\% fetal bovine serum (Gibco). For the cell experiments, the cells were cultured in serum free media containing $30 \mathrm{mM}$ glucose and $100 \mu \mathrm{M}$ palmitic acid for $24 \mathrm{~h}$.

\section{Cell transfection}

For knockdown experiments, three sequences of small interfering RNA (siRNA) targeting human FoxO3 or control non-silencing siRNA (Gene Pharma, China) were transfected into HepG2 cells for $48 \mathrm{~h}$. For overexpression experiments, adenovirus carrying green fluorescent protein (GFP) (Ad-GV314-GFP, GeneChem, China) or FoxO3 coding sequence (Ad-GV314-FoxO3, GeneChem, China) was diluted in PBS and added to media according to the multiplicity of infection (MOI). Generally, HepG2 cells were infected with adenovirus at an MOI of 50 for $24 \mathrm{~h}$. HepG2 cells were transfected with a plasmid expressing green fluorescent protein (GFP), or human FoxO3 (FoxO3-WT, \#1787, Addgene, USA), or human FoxO3 with three mutated phosphorylation sites (T32, S253, and S315) (FoxO3-TM, \#1788, Addgene, USA). 


\section{Oil red 0 staining}

The cells were washed with PBS twice and fixed with $10 \%$ paraformaldehyde for $30 \mathrm{~min}$. Oil red $\mathrm{O}$ staining was performed using an Oil red O staining kit (\#D027, Jiancheng Biotech, China) according to the manufacturer's protocols.

\section{Cellular triglyceride measurement}

Cells in 6-well plates were washed twice with PBS, and collected by scraping the cells. Cellular TGs were extracted using the chloroform/methanol $(2: 1 \mathrm{v} / \mathrm{v})$ method and dried in a chemical hood. After drying, $40 \mathrm{ul} 1 \%$ Triton X-100-ethol was added [19]. Then, the concentration of TG was measured using a TG reagent kit (Shensuo UNF, China) according to the manufacturer's protocols.

\section{Total RNA isolation and quantitative real-time PCR}

The total RNA of the cells and liver tissue were isolated using the TRIzol method. An RT reagent kit with cDNA eraser (Takara, Japan) was used to perform reverse transcription. SYBR Green Premix Ex Taq (Takara, Japan) was used to perform quantitative real-time PCR. The data were analyzed by the $2^{-\triangle \Delta C T}$ method. $\beta$-Actin was used as an internal reference. The primers are described in Table 1.

\section{Western blot analysis}

Protein extraction of cells and liver tissue was performed with RIPA in the presence of protease inhibitors. A BCA-100 Protein Quantitative Analysis Kit (Beyotime Biotechnology, China) was used to measure the protein concentration. A total of $20 \mu \mathrm{g}$ of protein lysates was loaded onto 10\% SDS-PAGE gels and transferred to polyvinylidene difluoride (PVDF) membranes (Millipore) and subjected to immunoblot analysis using antibodies against proteins.

\section{Dual luciferase reporter assay}

A luciferase reporter plasmid encoding the SREBP1c promoter of $-2000 /+194$ was constructed by the GeneChem Corporation (GeneChem, China). HepG2 cells were cotransfected with $0.5 \mu \mathrm{g}$ of empty vector or FoxO3 plasmid, along with $0.5 \mu \mathrm{g}$ of luciferase reporter plasmid and $50 \mathrm{ng}$ of pRL-CMV Renilla luciferase plasmid (Promega, USA) as an internal control in 12-well plates via lipo-3000 (Invitrogen) according to the manufacturer's instructions. After $48 \mathrm{~h}$, dual luciferase reporter assays were performed and analyzed according to the manufacturer's protocol.

Table 1 Sequence of primers for quantitative real-time PCR

\begin{tabular}{|c|c|c|c|}
\hline Gene & Species & Forward primer & Reverse primer \\
\hline FoxO3 & Human & TCAAGGATAAGGGCGACAGC & GGACCCGCATGAATCGACTA \\
\hline SREBP-1C & Human & GCGCCTTGACAGGTGAAGTC & GCCAGGGAAGTCACTGTCTTG \\
\hline SCD1 & Human & AGCTCATCGTCTGTGGAGCC & GCCACGTCGGGAATTATGAGG \\
\hline FAS & Human & GGGATGAACCAGACTGCGTG & TCTGCACTTGGTATTCTGGGT \\
\hline ACC1 & Human & ATGTCTGGCTTGCACCTAGTA & CCCCAAAGCGAGTAACAAATTCT \\
\hline CD36 & Human & CTITGGCTTAATGAGACTGGGAC & GCAACAAACATCACCACACCA \\
\hline MTTP & Human & ACAAGCTCACGTACTCCACTG & TCCTCCATAGTAAGGCCACATC \\
\hline PPARa & Human & TTCGCAATCCATCGGCGAG & CCACAGGATAAGTCACCGAGG \\
\hline$\beta$-actin & Human & GATGAGATTGGCATGGCTIT & GTCACCTTCACCGTTCCAGT \\
\hline FoxO3 & Mouse & CTCACTITGTCCCAGATCTACG & CTTCATTCTGAACGCGCATG \\
\hline SREBP-1C & Mouse & GTGAGCCTGACAAGCAATCA & GGTGCCTACAGAGCAAGAG \\
\hline ChREBP & Mouse & AGATGGAGAACCGACGTATCA & ACTGAGCGTGCTGACAAGTC \\
\hline SCD1 & Mouse & TTCTTGCGATACACTCTGGTGC & CGGGATTGAATGTTCTTGTCGT \\
\hline FAS & Mouse & GCTGCGGAAACTTCAGGAAAT & AGAGACGTGTCACTCCTGGACTT \\
\hline ACC1 & Mouse & ATGGGCGGAATGGTCTCTITC & TGGGGACCTTGTCTTCATCAT \\
\hline GPAM & Mouse & ACAGTTGGCACAATAGACGTIT & CСTTCCATTTCAGTGTTGCAGA \\
\hline DGAT2 & Mouse & GCGCTACTTCCGAGACTACTT & GGGCCTTATGCCAGGAAACT \\
\hline SREBP-2 & Mouse & GCAGCAACGGGACCATTCT & CCCCATGACTAAGTCCTTCAACT \\
\hline HMGCS1 & Mouse & AACTGGTGCAGAAATCTCTAGC & GGTTGAATAGCTCAGAACTAGCC \\
\hline HMGCR & Mouse & AGCTTGCCCGAATTGTATGTG & TCTGTTGTGAACCATGTGACTTC \\
\hline 36B4 & Mouse & AGATTCGGGATATGCTGTTGGC & TCGGGTCCTAGACCAGTGTTC \\
\hline
\end{tabular}




\section{Animal experiments}

We used 9-week-old C57BL/6 J male mice (Shanghai SLAC Laboratory Animal Company) for the chow diet study. We delivered recombinant adenovirus encoding FoxO3-cDNA or control adenovirus by tail vein injection in a total volume of $200 \mu$ l. The total viral load was approximately $3 \times 10^{9}$ plaque forming units per mouse. Each animal had free access to drinking water and chow and was housed at constant room temperature $(20 \pm 2)$ ${ }^{\circ} \mathrm{C}$ under a 12-h light/dark cycle. All animal experiments were approved by the Animal Use and Care Committee of Fudan University.

We performed the intraperitoneal glucose tolerance tests (IPGTT) on day 7 post-injection and intraperitoneal insulin tolerance tests (IPITT) on day 12 post-injection. On day 19 post-injection, mice were sacrificed after fasting overnight. Blood was collected from the eyeball and centrifuged at $3500 \mathrm{rpm}$ for $15 \mathrm{~min}$ at $4{ }^{\circ} \mathrm{C}$. For the obtained liver tissue, some of it was fixed in $10 \%$ formaldehyde and analyzed by H\&E staining, part was frozen with Tissue OCT-Freeze Medium (Sakura Finetek, USA) for Oil red O staining to evaluate hepatic TG concentration, and the remaining portion was placed in liquid nitrogen for protein, mRNA and TG analysis. Liver H\&E staining and Oil red O staining were performed by Wuhan Seville Biotechnology, China. The lipid profiles were detected by Shanghai BioTNT Company.

For the high-fat diet experiment, 8-week-old C57BL/6 J male mice were fed a high-fat diet (D12492, Research Diets, USA) for 20 days, with virus injection at a concentration of $2 \times 10^{9}$ plaque forming units per mouse on day 5 . We performed IPGTT on day 7 and IPITT on day 12 postinjection. After day 15 post-injection, mice were sacrificed.

$\mathrm{db} / \mathrm{db}$ mice, ob/ob mice and high glucose and high sucrose (HFHS) fed mice were gifted by Prof. Yu Li and Prof. Xin Gao.

\section{Intraperitoneal glucose tolerance tests}

Mice were fasted overnight before the experiment and injected intraperitoneally with glucose solution at a dose of $2 \mathrm{~g} / \mathrm{kg}$ body weight. Blood glucose was measured using a blood glucose meter (ROCHE, Germany) before and $15 \mathrm{~min}, 30 \mathrm{~min}, 60 \mathrm{~min}$, and $120 \mathrm{~min}$ post-injection.

\section{Intraperitoneal insulin tolerance tests}

Mice were fasted for $4-6 \mathrm{~h}$ before the experiment and injected intraperitoneally with regular human insulin at a dose of $1 \mathrm{U} / \mathrm{kg}$ body weight. Blood glucose was measured using a blood glucose meter (ROCHE, Germany) before and $15 \mathrm{~min}, 30 \mathrm{~min}, 60 \mathrm{~min}$, and $120 \mathrm{~min}$ post-infusion.

\section{Quantification of hepatic lipid content}

A total of $20 \mathrm{mg}-40 \mathrm{mg}$ of liver tissue was cut and homogenized in $1 \mathrm{ml}$ of PBS. The lipids in the tissue were extracted via the chloroform/methanol $(2: 1 \mathrm{v} / \mathrm{v})$ method and dried in a chemical hood. After drying, $200 \mu \mathrm{l}$ of ethanol was added to dissolve it. The concentration of TG was measured using a TG reagent kit (Shensuo UNF, China) according to the manufacturer's protocols.

\section{Statistics}

All data are presented as the mean \pm standard error of the mean (SEM). An unpaired two-tailed $t$ test or MannWhitney test was performed for two-group comparisons. For more than two groups, one-way ANOVA was performed for intergroup comparisons. The Chi-square test or Fisher's exact test was performed for the analysis of contingency tables. All data were analyzed with GraphPad Prism 7. $P<0.05$ was considered statistically significant.

\section{Results}

FoxO3 expression is elevated in the liver of NAFLD models and NAFLD patients

To determine whether there is a correlation between FoxO3 and NAFLD, we examined the expression of FoxO3 in the liver of different types of NAFLD models. According to the western blotting, the protein level of FoxO3 was upregulated in the livers of high fat and high sucrose (HFHS)-fed mice, ob/ob mice and $\mathrm{db} / \mathrm{db}$ mice compared with the chow diet mice or wild-type (WT) mice (Fig. 1a, c, e). The mRNA level of FoxO3 was also increased in the livers of HFHS mice and ob/ob mice, of which it was significantly increased in the livers of $\mathrm{db} / \mathrm{db}$ mice $(P=0.082)$ (Fig. 1b, d, f). Additionally, FoxO3 protein expression was increased dramatically in the livers of NAFLD patients (Fig. 1g). Next, we performed an analysis of FoxO3 protein in vitro. HepG2 cells were exposed to high glucose $(30 \mathrm{mM})$ and high palmitic acid $(100 \mu \mathrm{M}, \mathrm{PA})$ for $24 \mathrm{~h}$ to mimic the pathology of NAFLD. Consistently, the protein level of FoxO3 was increased dramatically in HepG2 cells challenged with high glucose and high palmitic acid (HFHG) (Fig. 1h). Additionally, the content of nuclear FoxO3 protein was also increased compared to that of the control group (Additional file 2: Figure S2a). These results indicate that FoxO3 expression becomes abnormally higher in fatty liver and steatotic cells, suggesting a close relationship between NAFLD and FoxO3.

\section{FoxO3 loss-of-function inhibits triglyceride accumulation in HepG2 cells}

To study the physiological effect of FoxO3 on hepatic TG metabolism, we used siRNA to knock down FoxO3 in HepG2 cells. FoxO3 siRNA1 and siRNA2 transfection triggered a significant decrease in FoxO3 protein levels compared to that of the control group (Fig. 2a). HFHG exposure induced higher levels of lipid droplets in HepG2 cells, which were markedly diminished by FoxO3 


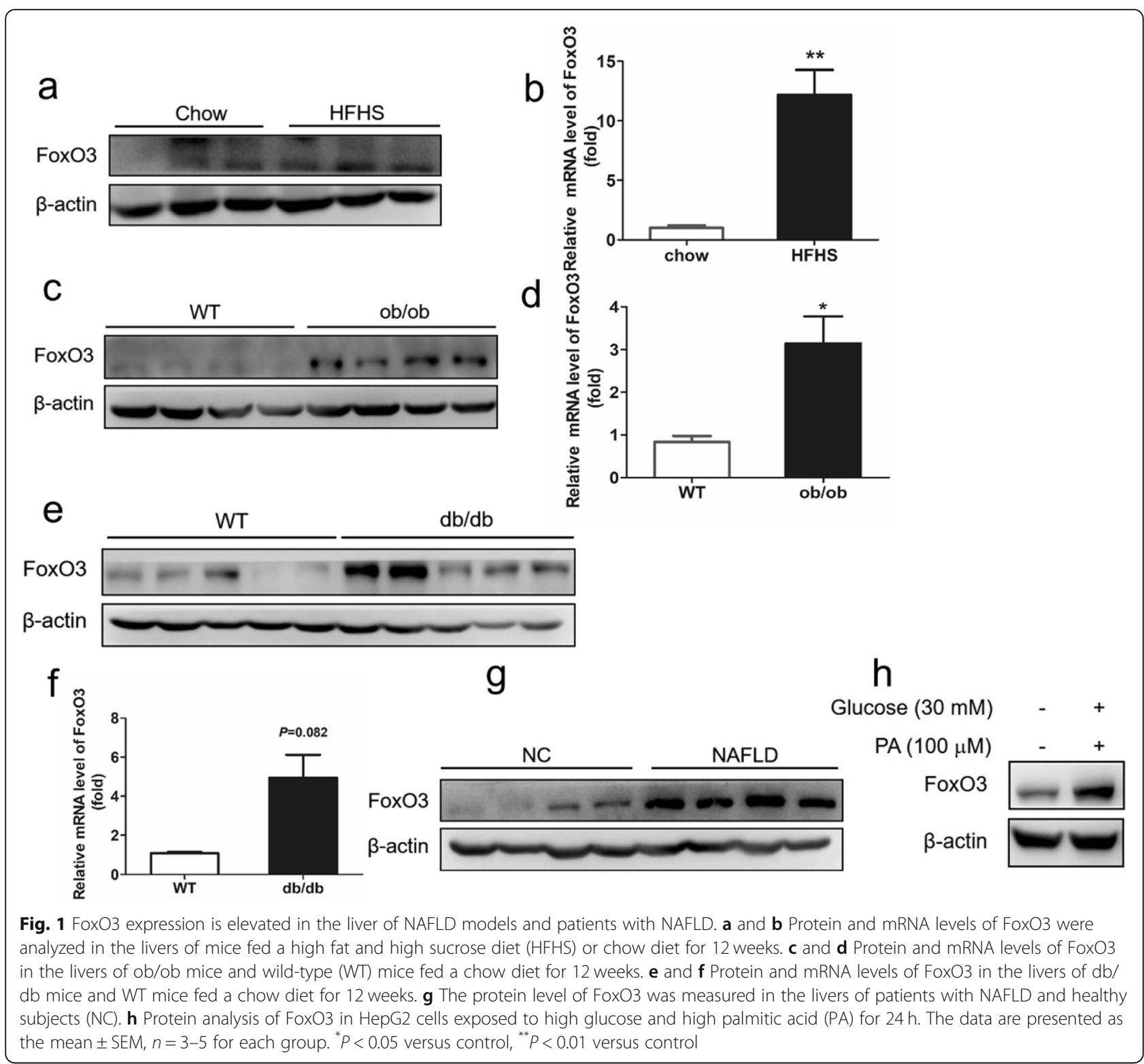

siRNA-transfection, as shown by Oil red $O$ staining (Fig. 2b). Quantitatively, the intracellular TG concentration was increased by HFHG administration and was decreased by FoxO3 siRNA treatment (Fig. 2c). These data imply that FoxO3 loss-of-function protects against hepatic steatosis induced by HFHG in vitro.

\section{FoxO3 gain-of-function results in hepatic steatosis in vitro} and in vivo

Next, we assessed the role of FoxO3 in TG homeostasis with gain-of-function approaches in the culture of hepatocytes and liver of mice. Overexpression of FoxO3 was achieved by adenovirus infection of HepG2 cells and was confirmed by western blotting (Fig. 3a). FoxO3 overexpression adenovirus (Ad-FoxO3) infection induced increased lipid droplets in HepG2 cells, as shown by Oil red $\mathrm{O}$ staining (Fig. 3b). Consistently, cellular TG concentration analysis suggested a trend toward an increase in Ad-FoxO3-treated cells (Fig. 3c). These data indicate a steatosis effect of FoxO3 on hepatocytes.

To confirm the effect of FoxO3 gain-of-function on hepatic steatosis, we injected adenovirus into adult mice via the tail vein. Ad-FoxO3 injection caused an upper shift in FoxO3 protein (Fig. 3d). Histological analysis of liver sections by Oil red $\mathrm{O}$ staining showed a high abundance of lipid droplets dispersed in Ad-FoxO3-treated mice compared to Ad-GFP-treated mice (Fig. 3e). The histological result was confirmed by the quantification analysis of hepatic TG content. Ad-FoxO3-treated mice showed 2-fold higher levels of hepatic TG content 


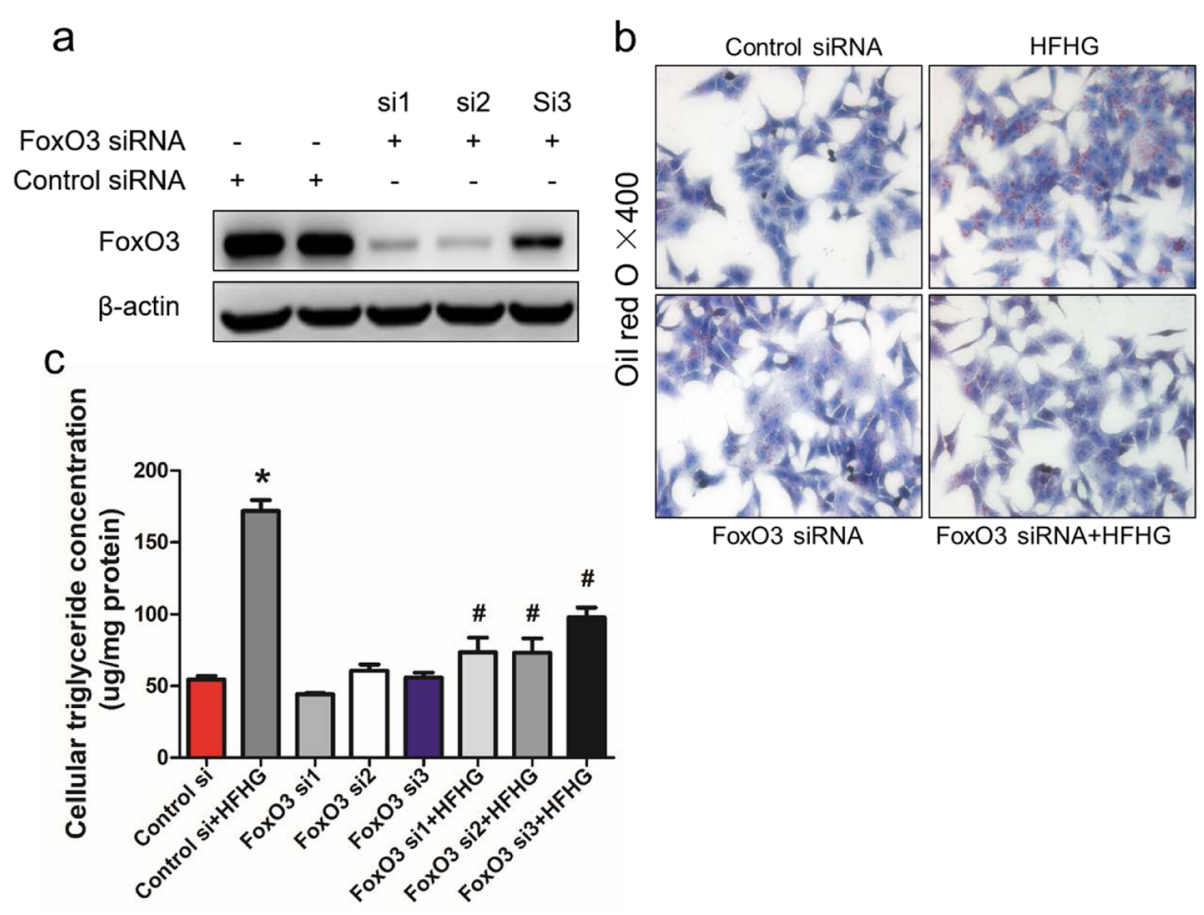

Fig. 2 FoxO3 loss-of-function inhibits triglyceride accumulation in HepG2 cells. a Protein analysis of FoxO3 in HepG2 cells transduced with three small interfering RNAs (FoxO3 si1, si2, and si3) or a negative control for $24 \mathrm{~h}$. b Representative morphology of Oil red O staining of HepG2 cells treated with FoxO3 siRNA1 or negative control for $24 \mathrm{~h}$ and then challenged with or without high glucose and high palmitic acid (HFHG) for another $24 \mathrm{~h}$. c Analysis of cellular TG concentrations of HepG2 cells transduced with FoxO3 si1, si2, si3 or negative control for $24 \mathrm{~h}$ and treated with or without HFHG for another $24 \mathrm{~h}$. The data are presented as the means \pm SEM. ${ }^{*} P<0.05$ versus control, ${ }^{*} P<0.05$ versus HFHG

compared with levels of control littermates (Fig. 3f). To examine what contributed to the higher hepatic TG concentrations, we measured the expression of lipogenic genes. As expected, the liver of Ad-FoxO3-treated mice demonstrated increased gene transcript levels of SREBP1c and its target genes (SCD1) (Fig. 3g). Other genes involved in TG synthesis, such as glycerol-3-phosphate acyltransferase (GPAM), which catalyzes the first committed step of TG synthesis [20], were highly expressed, whereas diacylglycerol acyltransferase 2 (DGAT2), which catalyzes the final committed step of TG synthesis [20], had no significant change (Fig. 3g). FoxO1 has been shown to be an important regulator of lipid metabolism [21]. To determine the potential effect of FoxO3 overexpression on the expression of FoxO1, we detected the mRNA level of FoxO1 and found no significant difference (Fig. 3g).

Carbohydrate response element binding protein (ChREBP), another transcription factor associated with glucose and lipid metabolism [22], was highly expressed in Ad-FoxO3treated-mice, but did not reach a significant difference (Additional file 3: Figure S3a). Additionally, the mRNA abundance of genes involved in cholesterol synthesis, including sterol regulatory-element binding protein 2 (SREBP-2) [23], 3-hydroxy-3-methylglutaryl-coenzyme A (HMGC) synthase 1 (HMGCS1) and HMGC reductase (HMGCR), had a tendency to increase but only that of SREBP-2 had a significant difference (Additional file 3: Figure S3a).

We also assessed the role of FoxO3 overexpression in glucose metabolism. In response to glucose injection, Ad-FoxO3-treated mice displayed elevated blood glucose levels at $30 \mathrm{~min}, 60 \mathrm{~min}$ and $120 \mathrm{~min}$ post-injection, in accordance with an increase in the area under the curve (AUC) (Fig. 3h and i). After insulin injection, the blood glucose concentrations demonstrated no significant difference between the two groups, with a mild increase in the AUC in Ad-FoxO3-treated mice (Fig. 3j and k). Consistently, Ad-FoxO3 treatment increased the gene expression of glucose-6-phosphatase (G6pc) and phosphoenolpyruvate carboxykinase1 (Pck1), two enzymes catalyzing the rate-limiting step of gluconeogenesis (Fig. 3g). No significant difference in body weight was observed (Fig. 3l).

Together, these data indicate that FoxO3 overexpression results in hepatic steatosis and impaired glucose tolerance.

\section{FoxO3 gain-of-function aggravates hepatic steatosis in mice fed a high-fat diet}

To assess the contribution of FoxO3 to TG accumulation in the pathological state, we next delivered adenovirus encoding FoxO3-cDNA to mice fed a high-fat diet. 


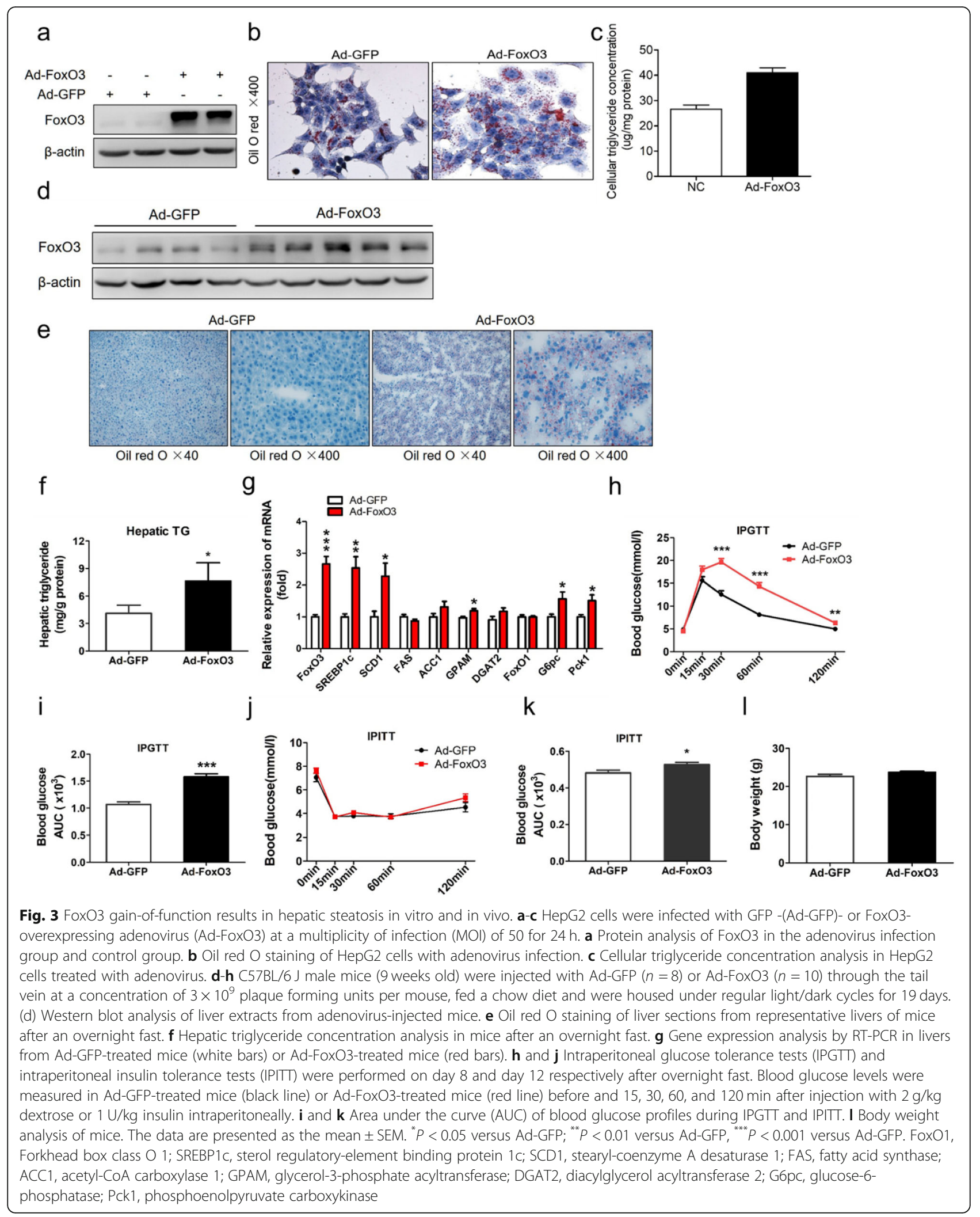




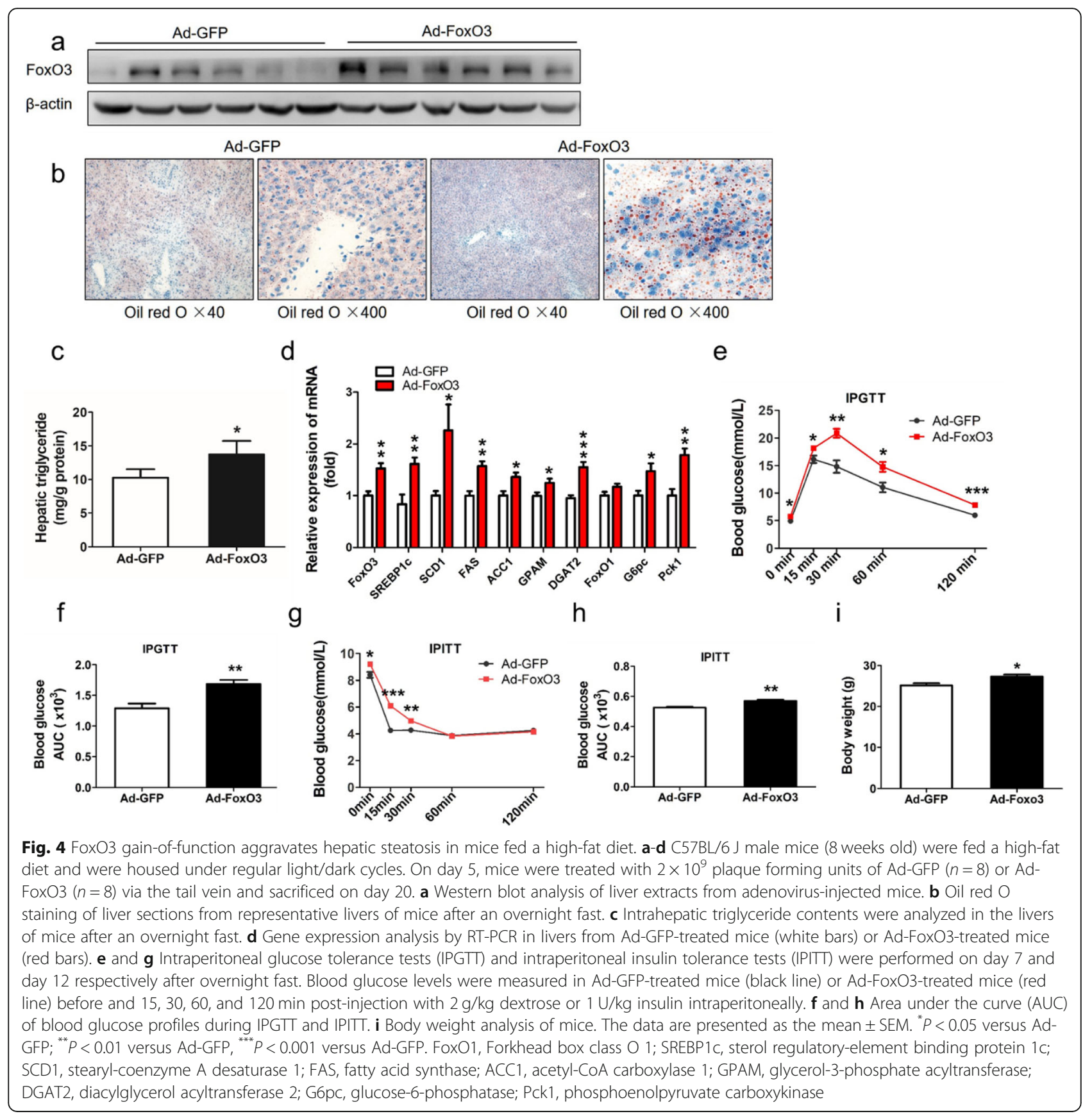

Ad-FoxO3-treated-mice showed elevated expression of FoxO3 protein (Fig. 4a). Histologically, Ad-FoxO3treated mice experienced numerous small and dense lipid droplets, whereas Ad-GFP-treated mice had little lipid droplets (Fig. 4b). Hepatic TG concentrations were also increased in Ad-FoxO3-treated mice (Fig. 4c). Consistently, the mRNA levels of SREBP1c and downstream target genes (SCD1, FAS, and ACC1) were increased, and the expression of genes involved in TG synthesis (GPAM and DGAT2) was increased markedly (Fig. 4d).
Consistently, the mRNA expression of FoxO1 showed no significant change (Fig. 4d). The mRNA expression of ChREBP, HMGCS1 and HMGCR was upregulated significantly relative to that of the control (Additional file 3: Figure S3b).

In the glucose tolerance tests, Ad-FoxO3-treated mice exhibited higher levels of blood glucose and increased AUC (Fig. 4e and f). Moreover, insulin tolerance tests revealed that Ad-FoxO3-treated mice showed reduced insulin tolerance at the set point of 
time of glucose measurements, as determined by an increase in AUC (Fig. 4g and h). Additionally, the mRNA levels of G6pc and Pck1 were also increased (Fig. 4d). Ad-FoxO3-treated mice had more body weight gain than control mice (Fig. 4i).

Together, these data indicate that FoxO3 overexpression aggravates hepatic steatosis and reduces glucose tolerance and insulin sensitivity in mice fed a high-fat diet.

\section{FoxO3 transcriptionally stimulates the expression of SREBP1c}

To define the underlying mechanism by which FoxO3 functions in hepatic steatosis, we transfected siRNA into HepG2 cells for knockdown of FoxO3 and examined the mRNA levels of genes involved in hepatic TG metabolism. Hepatic TG homeostasis is controlled by at least four pathways including free fatty acid uptake, fatty acid oxidation, de novo lipogenesis (DNL) and TG secretion. Fatty acid transmembrane transporter (CD36), peroxisome proliferator-activated receptor alpha (PPAR $\alpha$ ), SREBP1c and microsomal triglyceride transfer protein (MTTP) are the key regulators [24]. Interestingly, FoxO3 knockdown curbed the transcription and mRNA levels of SREBP1c and its downstream target genes (SCD1, FAS, and ACC1), without significantly influencing CD36, PPAR $\alpha$ and MTTP expression (Fig. 5a). FoxO3 downregulation by siRNA resulted in a marked repression of SREBP1c and SCD1 protein expression (Fig. 5b). FoxO3 overexpression mediated by plasmid increased the mRNA level of SREBP1c, without influencing the mRNA levels of CD36, PPAR $\alpha$ and MTTP (Fig. 5c). Plasmid-mediated overexpression of FoxO3 increased the protein levels of SREBP1c and SCD1 (Fig. 5d). Additionally, an induction of SREBP1c, in a manner that paralleled the increase in FoxO3 expression, was observed in HepG2 cells infected with Ad-FoxO3 (Fig. 5e). These observations imply that FoxO3 mediates the expression of SREBP1c.

FoxO3 has been shown to act as a transcriptional activator to regulate target gene expression [25]. To gain additional insight into the molecular mechanisms underlying the induction of SREBP1c by FoxO3, we analyzed the promoter of mouse and human SREBP1c genes and identified a novel FoxO3 binding site using the JASPAR database (http://jaspar.genereg.net/cgi-bin/jaspar_db.pl) (Additional file 4: Figure S4a and b). To confirm this mechanism, we performed a dual-luciferase reporter assay to detect the capacity of FoxO3 to activate the promoter of SREBP1c in HepG2 cells. FoxO3-TM cotransfection resulted in a two-fold induction of luciferase activity, equivalent to the increased activity induced by plasmid expressing LXR (Fig. 5f). These data indicate that FoxO3 transcriptionally activates the activity of the SREBP1c promoter.

\section{Discussion}

In this work, we characterized the role of FoxO3 in hepatic TG deposition in vitro and in vivo. We showed that knockdown or overexpression of FoxO3 reduced or increased lipid droplets in HepG2 cells. In vivo, FoxO3 gain of function increased TG accumulation, along with up-regulation of numerous lipogenic genes (SREBP1c, SCD1, FAS, ACC1, GPAM and DGAT2). Mechanically, FoxO3 promotes the transcriptional activity of the SREBP1c promoter.

In this study, we confirmed the abnormally higher expression of FoxO3 in various steatosis cell models, animal models and patients with NAFLD, implying a close link between FoxO3 and fatty liver. Further, FoxO3 loss of function via siRNA abolished the lipid accumulation induced by HFHG treatment in HepG2 cells, and overexpression of FoxO3 mimicked the effect of HFHG. In vivo, FoxO3 gain of function prompted hepatic TG deposition and impaired glucose tolerance on a chow diet, and those impacts were further enhanced when fed a high-fat diet. These results indicate that FoxO3 stimulates lipid accumulation in vitro and in vivo.

Current studies have highlighted the importance of FoxOs, especially FoxO1, in hepatic lipid metabolism using liver-specific triple-null mice or overexpression models. Combined ablation of FoxOs studies exhibited changes in the hepatic lipid content [12, 26, 27], whereas FoxO1 gain-of-function studies seemed to imply both positive and negative effects on lipid production and accumulation. For overexpression of FoxO1 in vivo, there are two studies showing increased hepatic TG $[28,29]$, one showing no change [30], and one showing decreased hepatic TG [31]. Together, FoxO1 is involved in liver lipid metabolism but the exact effects are conflicting. To our knowledge, there is only one study about FoxO3 loss or gain of function on hepatic TG deposition [12]. In this study, FoxO3 ablation exerted no impact on liver histopathology, but increased the gene transcriptional level of FAS in vivo. In vitro, FoxO3 gain of function decreased the mRNA level of FAS [12]. In contrast, we detected an increase in FAS mRNA levels in Ad-FoxO3injected mice. This discrepancy may be due to the differences in the level and model of FoxO3 overexpression, of which in their experiment, it was by 1500 -fold mediated by plasmid, and in our study, it was 100 -fold mediated by adenovirus. Moreover, the expression of FoxO1 remained unchanged with FoxO3 gain-of-function or knockdown in vitro and in vivo, excluding the interference of FoxO1 on lipid metabolism. Our study is the first to clarify that FoxO3 prompted hepatic TG deposition in vitro and in vivo.

The effect of FoxO3 on cholesterol metabolism has been poorly explored. Only one study reported that FoxO3 and sirt6 reduced LDL-cholesterol levels through 
a

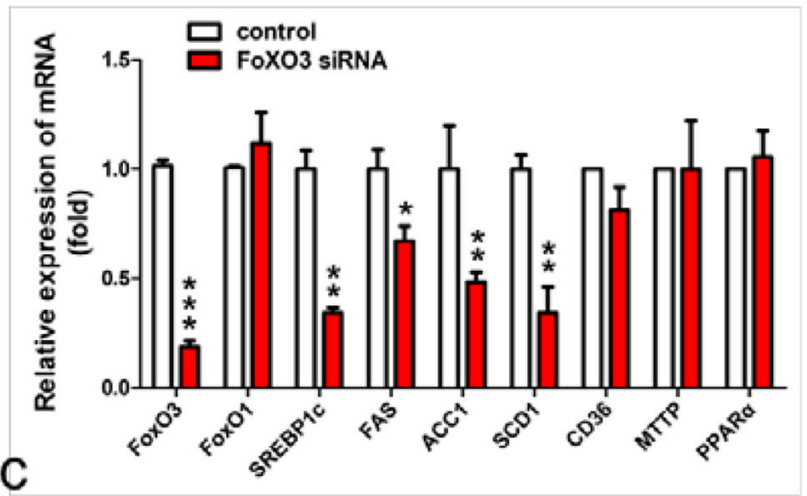

C

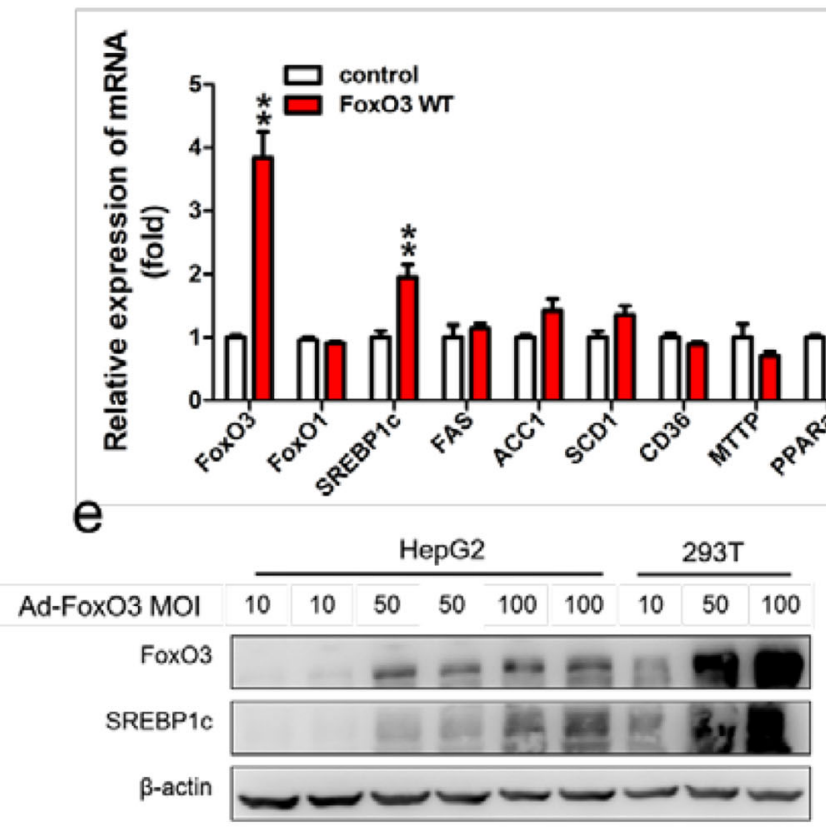

b

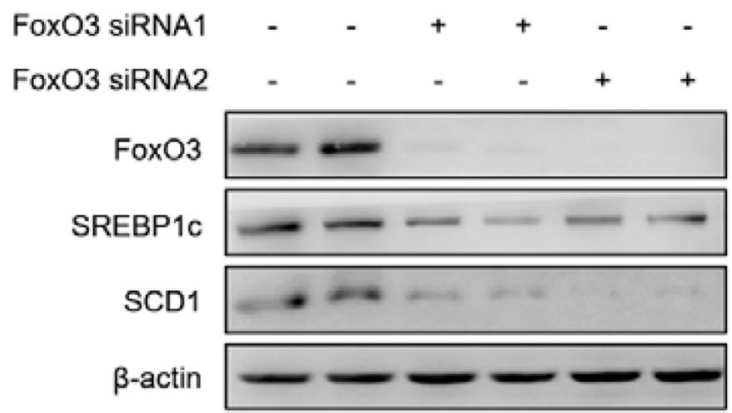

d

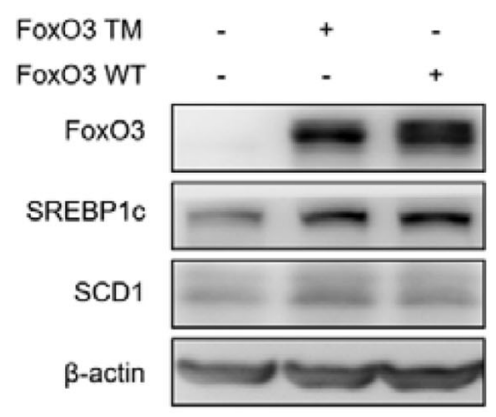

f

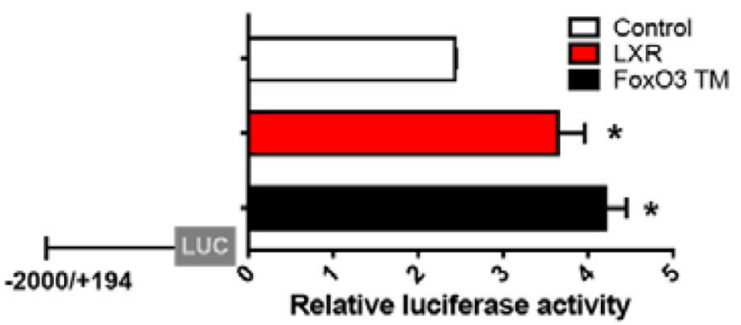

Fig. 5 FoxO3 transcriptionally stimulates the expression of SREBP1c. a Gene expression analysis of HepG2 cells transduced with FoxO3 siRNA or control siRNA for $48 \mathrm{~h}$. $\mathbf{b}$ The protein levels of FoxO3, SREBP1c and SCD1 transfected with FoxO3 siRNA or control siRNA. c Gene expression analysis of HepG2 cells transfected with plasmid. d The protein levels of FoxO3, SREBP1c and SCD1 in HepG2 cells transfected with plasmid. e FoxO3 and SREBP1c protein levels of HepG2 cells treated with adenovirus at different multiplicities of infection (MOls) for $24 \mathrm{~h}$. $\mathbf{f}$ Luciferase (Luc) reporter assays using constructs of the full-length (-2000/+ 194) SREBP1c gene promoter of mouse. Luc- SREBP1c was co-transfected into HepG2 cells for $24 \mathrm{~h}$ with a plasmid vector expressing the constitutively activated form of FoxO3 (FoxO3-TM) or an empty vector or plasmid expressing LXR. ${ }^{*} P<0.05$ versus control, ${ }^{* *} P<0.01$ versus control, ${ }^{* * *} P<0.001$ versus control. FoxO1, Forkhead box class O 1; SREBP1c, sterol regulatoryelement binding protein 1c; SCD1, stearyl-coenzyme A desaturase 1; FAS, fatty acid synthase; ACC1, acetyl-CoA carboxylase 1; CD36, fatty acid translocase protein; MTTP, microsomal triglyceride transfer protein; PPARa, peroxisome proliferator-activated receptor; LXR, liver X factor

regulation of the Pcsk9 gene [32]. Our study showed that hepatic FoxO3 overexpression led to increased expression of cholesterol synthesis genes (SREBP-2, HMGCR, and HMGCS1), indicating a potential effect of FoxO3 on cholesterol homeostasis that is worthy of further investigation.

Ad-FoxO3-treated mice displayed increased blood glucose concentrations after injection of glucose or insulin, indicating the impaired glucose tolerance and reduced insulin tolerance. This is in line with previous reports, showing that FoxO3 has a synergistic effect with FoxO1 on hepatic gluconeogenesis. Hepatic deletion of FoxO1 reduced fasting blood glucose concentrations by $20 \%$, and combined deletion of FoxO1 and FoxO3 further reduced fasting blood glucose concentrations by $40 \%$ [12]. Either FoxO1 or FoxO3 gain of function in vitro increased the gene expression of G6pc and Pck1 [12]. Additionally, intrahepatic TG accumulation also impairs insulin sensitivity and causes elevated blood glucose levels [33]. Thus, 
the reduced glucose tolerance and insulin tolerance in our study are likely due to the upregulated expression of gluconeogenic genes or the indirect effect of hepatic TG accumulation.

Our study points to a transcriptional stimulus of FoxO3 on SREBP1c gene expression to exert effects on liver histopathology. This conclusion is based on the following observations: 1) transfection with Foxo3 siRNA in HepG2 cells lowered the protein and mRNA levels of SREBP1c; 2) adenovirus or plasmid mediated overexpression of FoxO3 resulted in increased expression of SREBP1c; 3) SREBP1c expression was increased in the livers of Ad-FoxO3-treated mice; and 4) the constitutively active form of FoxO3 increased SREBP-1c promoter activity. The FoxO3-SREBP1c pathway is activated in over-nutrition states, as evidenced by the increased nuclear protein levels of FoxO3 and SREBP1c in our study. However, this pathway is inhibited under physiological conditions of inactivation of FoxO3 induced by insulin-dependent phosphorylation. Additionally, the mRNA expression of ChREBP, a glucose-activated transcription factor regulating glycolytic and lipogenic genes [22], was increased in the liver of Ad-FoxO3-treated mice, implying that activated SREBP1c may account partially for the hepatic lipid deposition in this study.

In the luciferase activity experiments, FoxO3-TM increased the Luc-SREBP1c luciferase activity by 2 -fold, equivalent to the increased activity observed in the positive control treated with LXR plasmid. LXR plays a key role in the transcriptional activation of SREBP1c [34]. This relatively lower stimulation may stem from the interference of endogenous SREBP1c activity. Endogenous SREBP1c binds to the SRE site on the SREBP1c promoter for feed-forward regulation [35], resulting in a relatively high luciferase activity in the control group. Moreover, a dual luciferase experiment implicates that FoxO3 activates the promoter of SREBP1c, and whether FoxO3 binds directly to the promoter of SREBP1c remains unknown. Studies have reported that there is no known FoxO-binding site in the proximal promoter of the SREBP1c promoter [36]. Moreover, studies showed that FoxO3 modulates gene expression via sequestering transcription factors and co-activators or binding to enhancers: FoxO3 recruits Sirt6 to the proximal promoter region of the proprotein convertase subtilisin/kexin type 9 gene to regulate cholesterol homeostasis [32]; FoxO3 binds to enhancers and increases polymerase II recruitment to active target genes [37]. Thus, we hypothesized that FoxO3 activation of the promoter of SREBP1c may not depend on direct promoter- binding. In conclusion, the underlying mechanism of how FoxO3 prompts SREBP1c gene expression should be investigated in further studies. Moreover, whether SREBP1c is required for the effect of FoxO3 on hepatic steatosis needs to be further explored.

\section{Conclusions}

The present study identifies that FoxO3 prompts hepatic triglyceride accumulation via transcriptionally stimulating the expression of SREBP1c. The FoxO3-SREBP1c pathway may play a potential role in the pathophysiology of NAFLD.

\section{Supplementary information}

Supplementary information accompanies this paper at https://doi.org/10. 1186/s12944-019-1132-2.

Additional file 1: Figure S1. FoxO3 expression was elevated in the livers of rats fed a high-fat diet. (a) Hepatic mRNA level of FoxO3. ${ }^{*} P<$ 0.05 vs. chow.

Additional file 2: Figure S2. FoxO3 was activated in HepG2 cells exposed to high glucose and high palmitic acid. (a) Nuclear protein of FoxO3 and SREBP1c in HepG2 cells exposed to high glucose and high palmitic (PA).

Additional file 3: Figure $\mathbf{5 3}$. The expression of ChREBP, SREBP-2, HMGCS1 and HMGCR was changed in the livers of mice injected with Ad-FoxO3. (a) mRNA levels of ChREBP, SREBP-2, HMGCS1 and HMGCR in the livers of mice treated with adenovirus fed a chow diet. (b) MRNA levels of ChREBP, SREBP-2, HMGCS1 and HMGCR in the livers of mice treated with adenovirus fed a high-fat diet (HFD). Ad-GFP, adenovirus expressing GFP; Ad-FoxO3, adenovirus expressing FoxO3. ${ }^{*} P<0.05$ vs. AdGFP, ${ }^{*} P<0.01$ vs. Ad-GFP.

Additional file 4: Figure S4. FoxO3 binding sites may exist in the promoter of SREBP1c. (a) The predicted FoxO3 binding sites in the promoter of the mouse SREBP1c gene using the JASPAR database. (b) The predicted FoxO3 binding sites in the promoter of the human SREBP1c gene using the JASPAR database.

\section{Abbreviations}

ACC1: Acetyl CoA carboxylase 1; Ad-FoxO3: FoxO3-overexpressing adenovirus; Ad-GFP: GFP-overexpressing adenovirus; AUC: Area under the curve; CD36: Fatty acid transmembrane transporter; ChREBP: Carbohydrate response element binding protein; DGAT2: Diacylglycerol acyltransferase 2; DNL: De novo lipogenesis; FAS: Fatty acid synthase; FoxO: Forkhead box class O; FoxO3-TM: Plasmid vector expressing the constitutively activated form of FoxO3; FoxO3-WT: Plasmid vector expressing FoxO3; G6pc: Glucose-6phosphatase; GPAM: Glycerol-3-phosphate acyltransferase; HFHG: High glucose and high palmitic acid; HMGCR: HMGC reductase; HMGCS1: 3hydroxy-3-methylglutaryl-coenzyme A (HMGC) synthase 1;

IPGTT: Intraperitoneal glucose tolerance tests; IPITT: Intraperitoneal insulin tolerance tests; LXR: Liver X receptor; MOI: Multiplicity of infection; mRNA: Messenger RNA; MTTP: Microsomal triglyceride transfer protein; NAFLD: Nonalcoholic fatty liver disease; PA: Palmitic acid;

Pck1: Phosphoenolpyruvate carboxykinase; PPARa: Peroxisome proliferatoractivated receptor alpha; PVDF: Polyvinylidene difluoride; RT-PCR: Real-time polymerase chain reaction; SCD1: Stearyl-coenzyme A desaturase 1; SEM: Standard error of the mean; siRNA: Small interfering RNA; SRE: Sterol regulatory element; SREBP1c: Sterol-regulatory element-binding protein 1c; SREBP-2: Sterol regulatory-element binding protein 2; TG: Triglyceride

\section{Acknowledgments}

We would like to acknowledge Prof. Xiao-yin Li from Fudan Institute for Metabolic Disease, Fudan University, Shanghai, for support and help in this study.

\section{Authors' contributions}

$H B, X G, H M Y$ and XPZ designed the experiments. LW, XPZ, XYS and XYY performed the experiments. LW, MFX and XXC analyzed and interpreted the data. LW and HB wrote the manuscript. All authors read and approved the final manuscript. 


\section{Funding}

This work was financially supported by the National Natural Science Foundation of China [81471073 to HB]; the National Key R\&D Program of China [2017YFC1309800 and 2017YFC1309801 to HB]; the Shanghai Science and Technology Committee [13441900303 to HB]; the Excellent Member Program of Zhongshan Hospital Fudan University [2015ZSYXGG1 to HB]; the Shanghai Municipal Population and Family Planning Commission [201740092 to HY]; and the Special Project of Integrating Traditional Chinese and Western Medicine in Shanghai General Hospital from the Shanghai Municipal Population and Family Planning Commission and Shanghai TCM Development Office [ZY (2018-2020)-FWTX-3019 to HY].

\section{Availability of data and materials}

All the data generated or analyzed during this study are included in this published article.

\section{Ethics approval and consent to participate}

Not applicable.

\section{Consent for publication}

Not applicable.

\section{Competing interests}

The authors declare that they have no competing interests.

\section{Author details}

${ }^{1}$ Department of Endocrinology and Metabolism, Zhongshan Hospital, Fudan University, Shanghai 200032, China. ${ }^{2}$ Fudan Institute for Metabolic Disease, Fudan University, Shanghai 200032, China.

Received: 4 April 2019 Accepted: 11 October 2019

Published online: 15 November 2019

\section{References}

1. Younossi Z, Anstee QM, Marietti M, Hardy T, Henry L, Eslam M, et al. Global burden of NAFLD and NASH: trends, predictions, risk factors and prevention. Nat Rev Gastro Hepat. 2017;15:11-20.

2. Byrne CD, Targher G. NAFLD: a multisystem disease. J Hepatol. 2014;62:S47-64.

3. Hardy T, Oakley F, Anstee QM, Day CP. Nonalcoholic fatty liver disease: pathogenesis and disease Spectrum. Annu Rev Pathol Mech Dis. 2016; 11:451-96.

4. Lambert JE, Ramos-Roman MA, Browning JD, Parks EJ. Increased De novo Lipogenesis is a distinct characteristic of individuals with nonalcoholic fatty liver disease. Gastroenterology. 2014;146:726-35.

5. Brent MM, Anand R, Marmorstein R. Structural basis for DNA recognition by FoxO1 and its regulation by posttranslational modification. Structure. 2008; 16:1407-16

6. Tia N, Singh AK, Pandey P, Azad CS, Chaudhary P, Gambhir IS. Role of Forkhead box O (FOXO) transcription factor in aging and diseases. Gene. 2018;648:97-105.

7. Kousteni S. FoxO1, the transcriptional chief of staff of energy metabolism. Bone. 2012;50:437-43.

8. Calnan DR, Brunet A. The FoxO code. Oncogene. 2008;27:2276-88.

9. Webb AE, Brunet A. FOXO transcription factors: key regulators of cellular quality control. Trends Biochem Sci. 2014;39:159-69.

10. Lee $\mathrm{S}$, Dong $\mathrm{HH}$. FoxO integration of insulin signaling with glucose and lipid metabolism. J Endocrinol. 2017:233:R67-79.

11. Tzivion G, Dobson M, Ramakrishnan G. FoxO transcription factors; regulation by AKT and 14-3-3 proteins. Biochim Biophys Acta. 1813;2011:1938-45.

12. Zhang K, Li L, Qi Y, Zhu X, Gan B, DePinho RA, et al. Hepatic suppression of Foxo1 and Foxo3 causes hypoglycemia and hyperlipidemia in mice. Endocrinology. 2012;153:631-46.

13. Amrit FRG, Steenkiste EM, Ratnappan R, Chen S, McClendon TB, Kostka D, et al. DAF-16 and TCER-1 facilitate adaptation to Germline loss by restoring lipid homeostasis and repressing reproductive physiology in C. elegans. PLoS Genet. 2016:12:e1005788.

14. Natarajan SK, Ingham SA, Mohr AM, Wehrkamp CJ, Ray A, Roy S, et al. Saturated free fatty acids induce cholangiocyte lipoapoptosis. Hepatology. 2014;60:1942-56.
15. Barreyro FJ, Kobayashi S, Bronk SF, Werneburg NW, Malhi H, Gores GJ. Transcriptional regulation of Bim by FoxO3A mediates hepatocyte Lipoapoptosis. J Biol Chem. 2007;282:27141-54.

16. Ragab S, Abdallah N, Hasan NS, Kandil ME, El Wasseif M, Elhosary Y, et al. FOXO 1a and FOXO 3a gene polymorphisms in association with metabolic syndrome. J Genet Eng Biotechnol. 2014;12:127-33.

17. Ferré P, Foufelle F. Hepatic steatosis: a role for de novo lipogenesis and the transcription factor SREBP-1c. Diabetes Obes Metab. 2010;12(Suppl 2):83-92.

18. Sanders FW, Griffin JL. De novo lipogenesis in the liver in health and disease: more than just a shunting yard for glucose. Biol Rev Camb Philos Soc. 2016:91:452-68

19. Zhu X, Yan H, Xia M, Chang X, Xu X, Wang L, et al. Metformin attenuates triglyceride accumulation in HepG2 cells through decreasing stearylcoenzyme a desaturase 1 expression. Lipids Health Dis. 2018;17.

20. Wang H, Airola MV, Reue K. How lipid droplets "TAG" along: Glycerolipid synthetic enzymes and lipid storage. Biochim Biophys Acta Mol Cell Biol Lipids. 1862;2017:1131-45.

21. Li Y, Ma Z, Jiang S, Hu W, Li T, Di S, et al. A global perspective on FOXO1 in lipid metabolism and lipid-related diseases. Prog Lipid Res. 2017:66:42-9.

22. Xu X, So JS, Park JG, Lee AH. Transcriptional control of hepatic lipid metabolism by SREBP and ChREBP. Semin Liver Dis. 2013;33:301-11.

23. Eberle D, Hegarty B, Bossard P, Ferre P, Foufelle F. SREBP transcription factors: master regulators of lipid homeostasis. Biochimie. 2004;86:839-48.

24. Kawano Y, Cohen DE. Mechanisms of hepatic triglyceride accumulation in non-alcoholic fatty liver disease. J Gastroenterol. 2013;48:434-41.

25. Eijkelenboom A, Mokry M, de Wit E, Smits LM, Polderman PE, van Triest $M$ et al. Genome-wide analysis of FOXO3 mediated transcription regulation through RNA polymerase II profiling. Mol Syst Biol. 2013;9:638.

26. Haeusler RA, Hartil K, Vaitheesvaran B, Arrieta-Cruz I, Knight CM, Cook JR, et al. Integrated control of hepatic lipogenesis versus glucose production requires FoxO transcription factors. Nat Commun. 2014;5190.

27. Tao R, Wei D, Gao H, Liu Y, DePinho RA, Dong XC. Hepatic FoxOs regulate lipid metabolism via modulation of expression of the Nicotinamide Phosphoribosyltransferase gene. J Biol Chem. 2011:14681-90.

28. Matsumoto M. Dual role of transcription factor FoxO1 in controlling hepatic insulin sensitivity and lipid metabolism. J Clin Invest. 2006;116:2464-72.

29. Qu S, Altomonte J, Perdomo G, He J, Fan Y, Kamagate A, et al. Aberrant forkhead box $\mathrm{O} 1$ function is associated with impaired hepatic metabolism. Endocrinology. 2006;147:5641-52

30. Zhang W, Patil S, Chauhan B, Guo S, Powell DR, Le J, et al. FoxO1 regulates multiple metabolic pathways in the liver. J Biol Chem. 2006;281:10105-17.

31. Zhang W, Bu SY, Mashek MT, O-Sullivan I, Sibai Z, Khan SA, et al. Integrated regulation of hepatic lipid and glucose metabolism by adipose triacylglycerol lipase and FoxO proteins. Cell Rep. 2016:15:349-59.

32. Tao R, Xiong X, DePinho RA, Deng C, Dong XC. FoxO3 transcription factor and Sirt6 Deacetylase regulate low density lipoprotein (LDL)-cholesterol homeostasis via control of the Proprotein Convertase Subtilisin/Kexin type 9 (Pcsk9) gene expression. J Biol Chem. 2013:288:29252-9.

33. Farese RV, Zechner R, Newgard CB, Walther TC. The problem of establishing relationships between hepatic Steatosis and hepatic insulin resistance. Cell Metab. 2012;15:570-3.

34. Chen GX, Liang GS, Ou JF, Goldstein JL, Brown MS. Central role for liver $X$ receptor in insulin-mediated activation of Srebp-1c transcription and stimulation of fatty acid synthesis in liver. P Natl Acad Sci Usa. 2004; 101:11245-50.

35. Amemiya-Kudo $M$, Shimano $H$, Yoshikawa $T$, Yahagi $N$, Hasty AH, Okazaki $H$, et al. Promoter analysis of the mouse sterol regulatory element-binding protein-1c gene. J Biol Chem. 2000;275:31078-85.

36. Deng $X$, Zhang W, O-Sullivan I, Williams JB, Dong O, Park EA, et al. FoxO1 inhibits sterol regulatory element-binding protein-1c (SREBP-1c) gene expression via transcription factors Sp1 and SREBP-1C. J Biol Chem. 2012; 287:20132-43.

37. Eijkelenboom A, Mokry M, Smits LM, Nieuwenhuis EE, Burgering BMT. FOXO3 selectively amplifies enhancer activity to establish target gene regulation. Cell Rep. 2013;5:1664-78.

\section{Publisher's Note}

Springer Nature remains neutral with regard to jurisdictional claims in published maps and institutional affiliations. 